\title{
Dielectric phase-transition and polarization studies in stepped and compositionally graded lead magnesium niobate-lead titanate relaxor thin films
}

\author{
R. Ranjith, Asis Sarkar, Apurba Laha, and S. B. Krupanidhi ${ }^{\text {a) }}$ \\ Materials Research Center, Indian Institute of Science, Bangalore 560012, India \\ A. K. Balamurugan, S. Rajagoplan, and A. K. Tyagi
Materials Science Division, Indira Gandhi Centre for Atomic Research, Kalpakkam 603102, India
}

Ferroelectric properties of chemically heterogeneous multilayers and graded composition thin films of $(1-x) \mathrm{Pb}\left(\mathrm{Mg}_{1 / 3} \mathrm{Nb}_{2 / 3}\right) \mathrm{O}_{3}-x \mathrm{PbTiO}_{3}$, a well-known relaxor, were prepared using a multitarget pulsed laser ablation system. Target materials with different values of $x(0.0,0.1,0.2$, and 0.3$)$ were used for ablation growth. Films with sharp interfaces and graded interfaces were prepared and were characterized for their electrical properties. The stepped films were found to have a phase-transition behavior dominated by the individual layer behavior with their corresponding local dielectric maxima. The graded films showed an overall averaged effect of the system. The graded films showed an enhanced diffusiveness in the phase transition and the $\gamma$ value was found to be 2.43, which is a measure of diffusivity. The tunability of these graded films was found to be high around $60 \%$ and was twice that of any of the film of homogeneous composition films. The room-temperature polarization hysteresis curves exhibited slim loop characteristics with a saturation polarization of about $40 \mu \mathrm{C} / \mathrm{cm}^{2}$ in the case of both stepped and graded films.

\section{INTRODUCTION}

Ferroelectric multilayers and thin films with compositional fluctuation across the film through thickness are recently well known for their striking properties that were not observed in conventional ferroelectric layers. ${ }^{1-3}$ The compositionally graded films are known to have a higher tunability, polarization, and flatter temperature characteristics because of their enhanced chemical heterogeneity. ${ }^{4}$ The (1 $-x) \mathrm{Pb}\left(\mathrm{Mg}_{1 / 3} \mathrm{Nb}_{2 / 3}\right) \mathrm{O}_{3}-x \mathrm{PbTiO}_{3}$ shortly known as PMNPT or PMNT is well known as a relaxor ferroelectric with a perovskite type of structure, which has been studied extensively with various compositions of PT and it has been proven as a promising material for solid-state actuators and multilayer capacitors. ${ }^{5}$ The $67 \%$ PMN and 33\% PT is known as the morphotropic phase boundary composition in the PMN-PT phase diagram and separates the rhombohedral phase of the system from the tetragonal phase. ${ }^{6}$ This composition is also found to have superior properties than other relaxor compositions. ${ }^{7,8}$ The characteristic properties of the relaxor ferroelectrics are a diffused phase transition, frequency-dependent dielectric maxima, frequencyindependent behavior of dielectric constant above the transition, slim loop hysteresis, a nonlinear dielectric response for bias voltages, and a local polarization at temperature above $T_{m}{ }^{9}$

The structural aspect of PMNPT relaxors has been studied extensively by various models such as space-charge model $^{10}$ and random site model. ${ }^{11}$ The presence of polar

\footnotetext{
a) Author to whom correspondence should be addressed; electronic mail: sbk@mrc.iisc.ernet.in
}

clusters showing domainlike characteristics with $\mathrm{Mg}$ and $\mathrm{Nb}$ associated with a local charge imbalance on a nonpolar matrix leading to a global charge balance and the presence of symmetry breaking within the system is widely accepted. ${ }^{12-14}$ The presence of these interactive polar clusters and their ergodic behavior above a certain freezing temperature was believed to be responsible for the diffusiveness in phase transition and also the existence of high saturation polarization values well above the transition temperature. ${ }^{9}$ Though the PMNPT relaxors were studied widely both experimentally and theoretically, there has been no unique theory that could explain the complete behavior of these materials. There are various models explaining the relaxor ferroelectrics, such as inhomogeneous microregion, ${ }^{15}$ superparaelectric with polarization fluctuation, ${ }^{9}$ dipolar glass, ${ }^{16,17}$ random field, ${ }^{18}$ interphase boundary motion, ${ }^{19}$ and spherical random-bond random-field model. ${ }^{20}$ The primary motive of this work is to enhance the chemical heterogeneity artificially, which in turn introduces a strain in the lattice, and effectively enhances the physical properties, with a dependence on the microscopic effects of the lattice. The approach of both compositionally varying multilayered systems and compositionally graded systems is well established for their local chemical heterogeneity and the lattice strain to give rise to improved physical properties superior to the properties observed in any other homogeneous compositions.

\section{EXPERIMENT}

$(1-x) \mathrm{Pb}\left(\mathrm{Mg}_{1 / 3} \mathrm{Nb}_{2 / 3}\right) \mathrm{O}_{3}-x \mathrm{PbTiO}_{3}$ ceramics were prepared through Coloumbite process ${ }^{21}$ with varying compositions of PT. The composition of PT content was varied from 
$30(x)$ at. \% to pure PMN ceramic. High dense ceramic pellets of different compositions, namely, $x=0.3,0.2,0.1$, and 0.0 of $12-\mathrm{mm}$ diameter and $4 \mathrm{~mm}$ thick were prepared. The crystallinity, composition, and the electrical behavior of the targets with homogeneous composition were studied and reported in the earlier studies. These pellets were used as the target materials for laser ablation. All the films used in this study were fabricated in a multitarget laser ablation chamber, and a $\mathrm{KrF}$ excimer laser of $248 \mathrm{~nm}$ (Lambda Physik) at $650{ }^{\circ} \mathrm{C}$ in an oxygen ambient of 100 mTorr with pulse energy of $160 \mathrm{~mJ} /$ pulse on $\langle 111\rangle$-oriented platinum-coated silicon substrates with the following sequence $\mathrm{Pt} / \mathrm{TiO}_{2} / \mathrm{SiO}_{2} / \mathrm{Si}$.

Thin films of these different compositions were prepared in three different configurations. (i) Thin films of individual compositions of single layers with homogeneous composition across the film through the thickness (for instance, the thin-film samples with PMN70-PT30 composition will be labeled as PMNT70-30 further, and pure PMN with zero PT will be labeled as PMN). (ii) The multilayers of these selected compositions were deposited one over the other in situ without breaking the vacuum, such that a sharp interface is maintained between each layers. The thin films were deposited in the following sequence, PMNT70-30/PMNT80-20/ PMNT90-10/PMN/LSCO/substrate. $\mathrm{La}_{0.5} \mathrm{Sr}_{0.5} \mathrm{CoO}_{3}$ (LSCO), a conducting oxide layer, was used as a template layer over platinum substrates. ${ }^{22}$ The films of this kind of stacking sequence were grown at $650{ }^{\circ} \mathrm{C}$ and were cooled down to room temperature in an oxygen ambient and the individual layer thickness was kept around $100 \mathrm{~nm}$. Films grown in these process conditions were expected to have a sharp interface among the different layers present and will be named as stepped films further. (iii) The third type of films grown was with the same stacking sequence and deposition conditions of that of the stepped films, with an additional postdeposition annealing at $600{ }^{\circ} \mathrm{C}$ for $20 \mathrm{~min}$, were carried out in an oxygen ambient of 100 mTorr. This was done to redistribute the composition of titanium, wherein this redistribution gives rise to a smooth composition gradient unlike the previous films with stepped composition. The postdeposition annealing also relieves the stress present at the interfaces and hence effectively, interdiffusion of the films is allowed to take place, which in turn broadens the interface. The details of the interface studies are dealt later in this article. These kinds of films with graded composition will be named as graded films further. The total thickness of all the films fabricated as a single layer, stepped, and graded films was kept constant, such that the electrical characteristics of these three films offer a meaningful comparison. The total thickness of these films was determined from secondary-ion-mass spectroscopy (SIMS) studies and was found to be around $0.45 \mu \mathrm{m}$. Thin films with a change in the direction of the stacking and gradient were also deposited but were not discussed in detail, because the nature of phase transition and polarization behavior with temperature was the same except a change in the asymmetry observed in the opposite direction. Since, the physical behavior was the same except for their magnitudes of physical entities, the characteristics of those films are not discussed in detail.

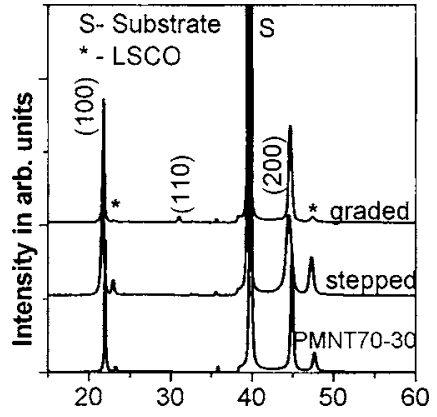

(a)

$2 \theta$ (degree)

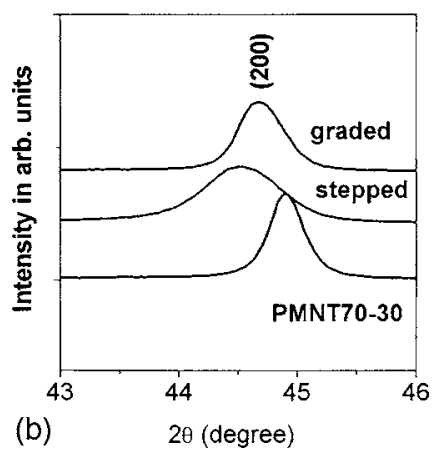

FIG. 1. (a) XRD pattern of the laser-ablated thin films (b) Magnified XRD pattern of (200) reflection of laser-ablated thin films.

The crystallinity of these films was cross-checked by means of $\mathrm{x}$-ray-diffraction pattern obtained using a Philips powder diffractometer. The sharpness of the interface and the composition gradient observed in the interfaces were studied using secondary-ion-mass spectrometer (Cameca 4f). The films with good crystallinity were used for further studies. A thin layer of gold was deposited over the top of the films by thermal evaporation in the form of circular dots with a diameter of $500 \mu \mathrm{m}$, by means of physical masking. These gold dots served as the top electrodes for the electrical characterization. Electrode annealing was done at $300{ }^{\circ} \mathrm{C}$ for $20 \mathrm{~min}$ for enhancing the adhesiveness of the electrodes with the surface of the films. The platinum surface of the substrate served as a bottom electrode and the gold dots acting as a top electrode, the samples were characterized in MIM configuration. The electrical characterizations such as frequency dispersion of the dielectric constant and capacitance-voltage $(C-V)$ behavior were carried out using an impedance analyzer of Agilent technologies (4294A). The polarization hysteresis $(P-E)$ measurements were carried out using RT66A operating in the Sawyer-Tower mode. ${ }^{23}$

\section{RESULTS AND DISCUSSION}

\section{A. X-ray diffraction}

The x-ray-diffraction pattern of the single layer of PMNT70-30, stepped, and graded films is shown in Fig. 1(a). The other compositions of single layers also showed exactly a similar kind of pattern and hence are not shown in the figure. The x-ray pattern revealed that the films are highly oriented along (100) direction irrespective of the nature of the compositional ingredient of the films for the specified process parameters in the fabrication process. Films were 

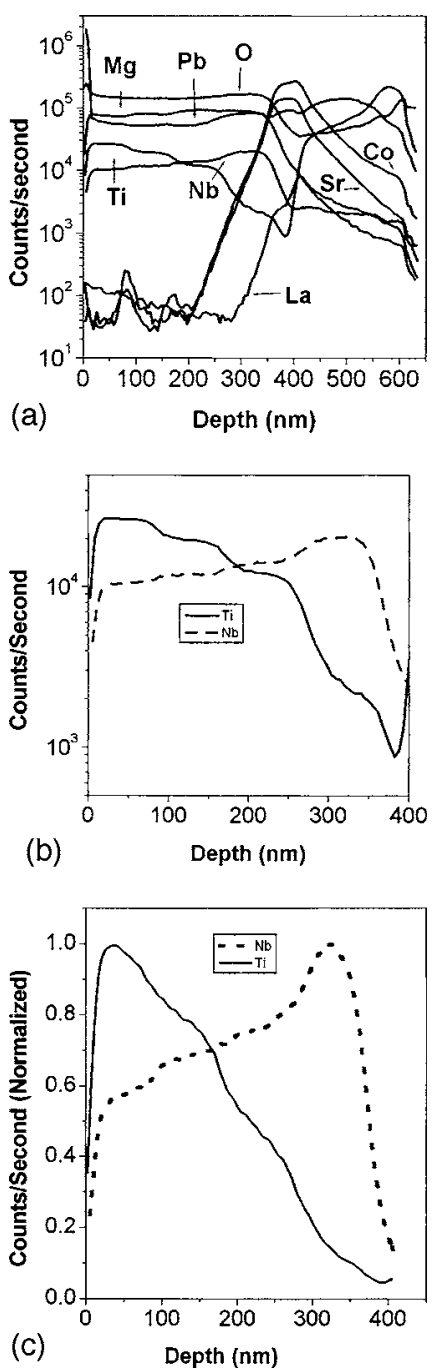

FIG. 2. (a) SIMS-based depth profile of a stepped film. (b) SIMS-based depth profile of $\mathrm{Ti}$ and $\mathrm{Nb}$ of a stepped film. (c) SIMS-based depth profile of $\mathrm{Ti}$ and $\mathrm{Nb}$ of a graded film.

also found to be absolutely free from pyrochlore phase, which is an unwanted cubic phase commonly observed in many of the lead-based systems. ${ }^{21}$ The broadening of the peaks was observed for the multilayers from that of the single layers and also a small shift in the theta value. This is due to the lattice strain present in the system, which arises due to mismatch in the lattice parameters of the individual layers on the increase of titanium concentration. The strain broadening of the x-ray peaks was found to be less in the case of graded films than that of the stepped films. Hence, postdeposition annealing has relaxed the strain at the interfaces by enhancing the interdiffusion of these films. The broadening of the (200) peaks is shown in Fig. 1(b).

\section{B. SIMS analysis}

Figure 2(a) shows the depth profile of a stepped film. Figures 2(b) and 2(c) show the depth profile of titanium (Ti) and niobium $(\mathrm{Nb})$ in both the stepped and graded films, respectively, observed through secondary-ion-mass spectrometer. Depth profiles of $\mathrm{Ti}$ and $\mathrm{Nb}$ are only shown to have a clear picture of the interfaces, whereas magnesium was
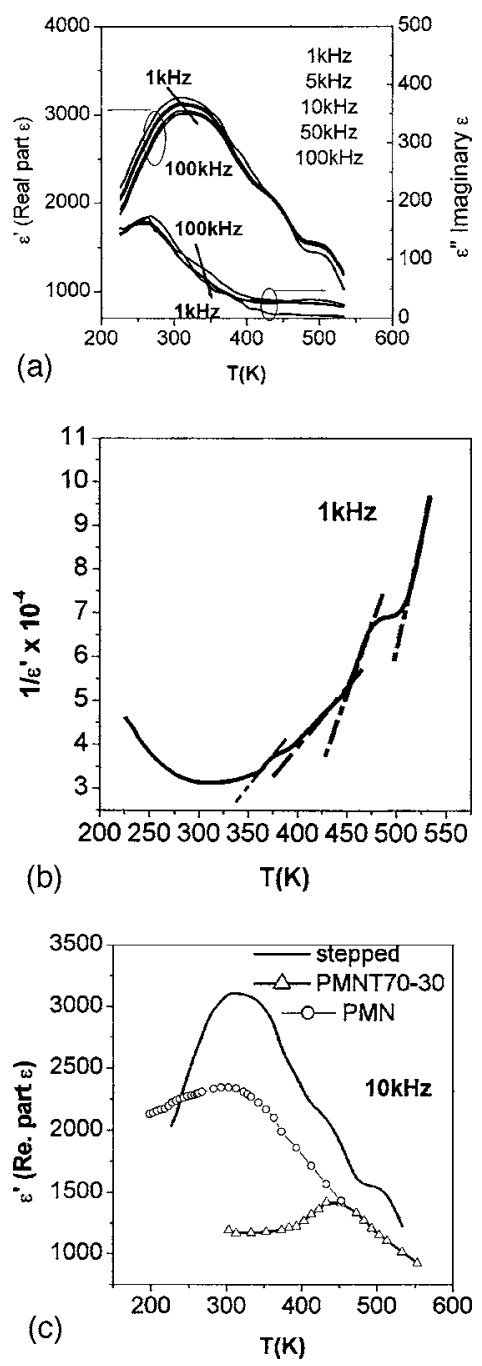

FIG. 3. (a) Dielectric phase transition of a stepped film. (b) Curie-Weiss plot of a stepped film. (c) Dielectric phase transition of a stepped film and the extreme single-layer compositions.

found to have the similar trend as of $\mathrm{Nb}$. The interface width of the stepped films between individual layers was found to be around $20 \mathrm{~nm}$. In the case of graded films the interface was found to be diffused and was around $60 \mathrm{~nm}$. Though the concentration gradient was not uniform throughout the film the interfaces were found to be broadened from that of the stepped films, which in turn relieves the lattice strain present at the interfaces of the stepped films. The SIMS studies clearly establish the stepped and graded variations of composition across the thickness. The rise of $\mathrm{Ti}$ concentration beyond the films is due to the presence of a $\mathrm{TiO}_{2}$ layer in the substrate configuration as explained earlier.

\section{Dielectric phase transition}

Dielectric phase transition of PMN-PT stepped and graded composition films are shown is Figs. 3(a) and 4(a), respectively. The phase-transition curves showed a very broad transition over a wide range of temperatures. In the case of stepped films the dielectric phase transition showed in Fig. 3(a) can be visualized as a combination of the following. ${ }^{24,25}$ The effective dielectric constant of the sys- 

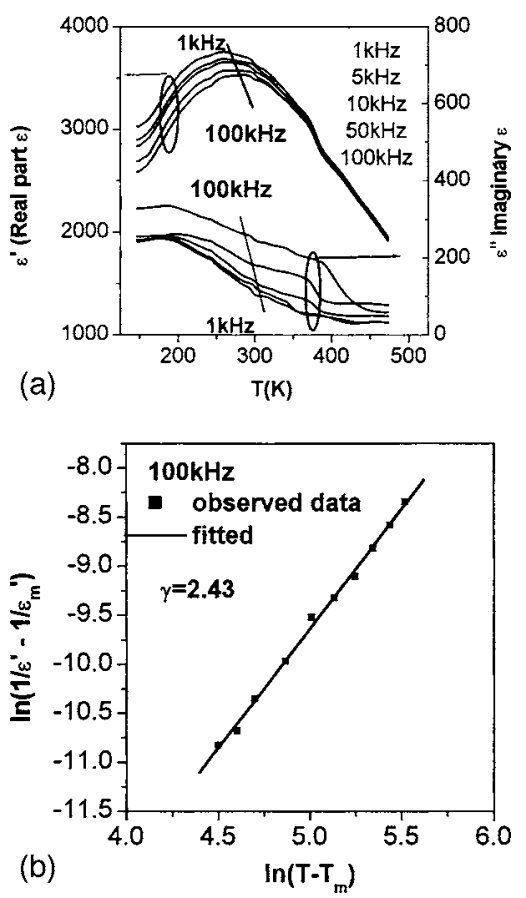

FIG. 4. (a) Dielectric phase transition of a graded film. (b) Modified CurieWeiss plot of a graded film.

tem, which is a function of the relaxation time of the polar clusters and temperature, can be written as follows:

$$
\varepsilon_{\text {eff }}=\sum_{i=1}^{4} \varepsilon_{i}\left(\tau_{i}, T_{i}\right)+\sum_{i<j}^{i=1 \rightarrow 3, j=2 \rightarrow 4} J_{i, j} \varepsilon_{i} \varepsilon_{j}+\varepsilon_{\text {int }} \text {, }
$$

where $\varepsilon_{\text {eff }}$ is the effective dielectric constant of the whole system and the first term in the above equation is a combination of the individual layers present in the stepped films and the second term is the interaction between the individual layers. $J$ is the interaction term between the two layers, though the interaction between all the layers is taken into account in the above equation, the interaction between the adjacent layers would be more significant than the interaction between the alternate layers. The interaction within the alternate layers may be significant when the thickness dependence of the individual layers is taken into account, which is not discussed in this paper. The suffix $i=1,2,3$, and 4 represents the dielectric constant of the pure PMN, PMNT9010, PMNT80-20, and PMNT70-30, respectively. The third term corresponds to the interfaces present in the system, which can be broadly classified as active and passive interfaces present in the system. The interface present between the LSCO layer and the relaxor material is known to have its own relaxation time but independent of temperature. ${ }^{26}$ The active interfaces are the interfaces present between the individual relaxor compositions with relaxation time and temperature dependence. A clear discontinuity or a stepped behavior is seen in the phase-transition curves with an increase of temperature in the case of stepped films. Four distinct local maxima were observed in the phase-transition curve that can be seen clearly from the normal Curie-Weiss plot shown in Fig. 3(b). The local linear regions after each local minimum were extrapolated to intersect the $x$ axis and the temperatures corresponding to those intercepts were found to be close to the $T_{m}$ of all the individual layers present. The local maxima observed in the phase-transition curve are found to be due to the domination of an individual layer at that temperature regime, which is clearly seen from Fig. 3(c). The figure shows the dielectric phase-transition curve of a stepped and the single layers with composition corresponding to the extreme layers of the stepped films at $10 \mathrm{kHz}$. This increase in the $T_{m}$ of the local maxima from that of the $T_{m}$ of the individual layers may be attributed to the stress present in the lattice ${ }^{27}$ and also due to the interaction of the polar clusters present with the successive layer. Hence, the stepped films can be considered to have an overall effect with a linear combination of all the factors explained above. Quantitative analysis of the interfaces and resolving the contributions of the individual components involve a rigorous theoretical calculation of the thermodynamic free energy of the system with the polarization of the individual layers and the interfaces, etc., which is under study. The dielectric phasetransition curve of the graded films shown in Fig. 4(a). can be visualized as an ensemble-averaged phenomenon of the polar clusters present. ${ }^{4}$ The phase-transition curve was found to be broad with single maxima and the $T_{m}$ was found to be an averaged value of the overall composition present. There was also a clear frequency dependence in the dielectric maxima $\left(\varepsilon_{m}\right)$ and the $T_{m}$. The phase transition is found to have a combinatorial effect of all the local compositional fluctuations present and the strain field, which were actually redistributed over the film by means of the postdeposition annealing of the films. The temperature and frequency dependences of the $\varepsilon_{\max }$ were not as prominent as in a normal single composition relaxor ferroelectric thin film. The $\varepsilon_{\max }$ was found to be constant for a small range of temperatures and also for a very narrow frequency band. In the case of normal relaxor ferroelectric, the temperature and frequency dependences of $\varepsilon_{\max }$ are attributed to the size distribution and relaxation times of the nanopolar clusters present in the system. $^{28}$

In the case of graded relaxor ferroelectric it is assumed that apart from the spatial variation of the composition and size of the clusters, the spatial fluctuation in the density of these polar clusters should also play a major role. Hence, the normal Vogel-Fultcher relationship used for the spin glass systems is generally obeyed by the homogeneous relaxor ferroelectric, which cannot be directly used in the case of graded ferroelectrics. The enhancement in the diffusiveness of the phase transition was cross-checked by means of using the modified Curie-Weiss plot which is generally used to quantify the extent of diffusiveness present in the film. The modified Curie-Weiss equation is given below.

$$
\left(\varepsilon^{\prime}-\varepsilon_{m}\right)=\frac{C}{\left(T-T_{m}\right)^{\gamma}},
$$

where $\varepsilon^{\prime}$ is the dielectric constant at a given temperature and $\varepsilon_{m}$ is the dielectric maxima. $T$ is the temperature, $T_{m}$ is the temperature corresponding to $\varepsilon_{m}, C$ is the Curie constant, and $\gamma$ is the parameter that quantifies the diffusivity of the system. In general $\gamma=2$ for a normal ferroelectric relaxor. The modified Curie-Weiss plot was plotted at temperatures 


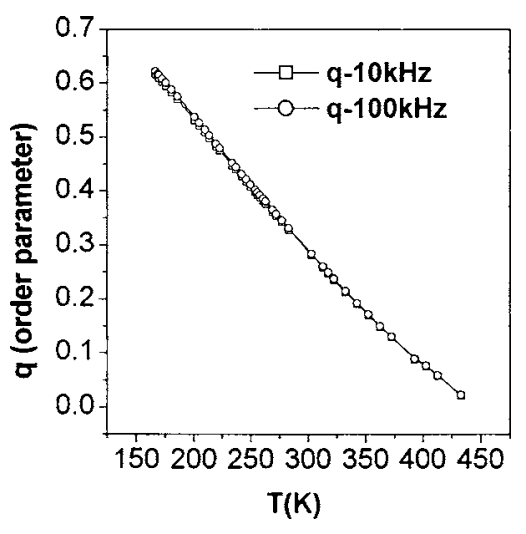

FIG. 5. Temperature dependence of the order parameter of a graded film.

well above the $T_{m}$ and in our case it was plotted in the range of $375-513 \mathrm{~K}$ whereas the $T_{m}$ was found to be near about $313 \mathrm{~K}$ at $100 \mathrm{kHz}$. The $\gamma$ value was determined from the linear fit of the plot between $\ln \left(1 / \varepsilon^{\prime}-1 / \varepsilon_{m}\right)$ and $\ln \left(T-T_{m}\right)$ and was found to be around 2.43 and the Curie constant was found to be $1.05 \times 10^{5}$ at $100 \mathrm{kHz}$ whose plot is shown in Fig. 4(b) and the $T_{C}$ value was found by extrapolation of the linear fit and was found to be $370 \mathrm{~K}$. The $\gamma$ value was found to be around 2.5 and the Curie constant was found to be $1.45 \times 10^{5}$ for $10 \mathrm{kHz}$ whose plot is not shown. A normal relaxor ferroelectric used to have quadratic dependence with temperature in the modified Curie-Weiss law, and this enhanced value of $\gamma$ from 2 can be used as a proof for the enhanced diffusiveness. Plotting a single modified CurieWeiss plot for the stepped films may not be meaningful to analyze the overall phase transition, because the phase transition is seen to be a combination of individual layer contributions at different temperatures.

The fraction of polar clusters responding at the temperatures both above and below $T_{m}$ is characterized by means of an entity called order parameter. The order parameter is expected to take a zero value after $T_{C}$, whereas in the case of normal ferroelectric relaxors the order parameter takes a nonzero value even for temperatures well above $T_{C}$, proving the existence of polar clusters above the $T_{C}$. The order parameter was calculated by the formula given below. ${ }^{29}$

$$
\varepsilon=\frac{C[1-q(T)]}{T-T_{C}[1-q(T)]} .
$$

$q(T)$ is the order parameter which quantifies the fraction of polar clusters existing above $T_{C}$. Though the above equation was given for in the case of single-layered films using the $T_{C}$ value of the graded film, it can be used in the graded or heterostructured films as well. ${ }^{26}$ The order parameter of the graded films was calculated at 10 and $100 \mathrm{kHz}$ and its temperature behavior is shown in Fig. 5. The value was found to be nonzero even at temperatures around $430 \mathrm{~K}$, which is well above the $T_{C}$. This shows the existence of polar clusters well above the transition temperature. The above equation cannot be used in the case of stepped films since they do not have a single transition temperature.
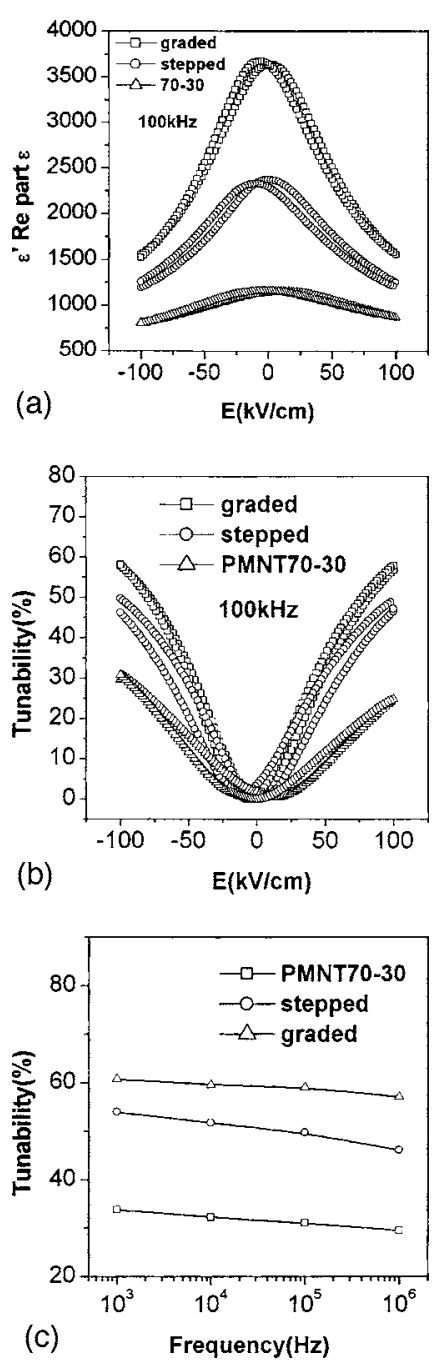

FIG. 6. (a) Nonlinear dielectric response of stepped, graded, and PMNT(70/30) compositions. (b) Tunability of stepped, graded, and PMNT70-30 compositions. (c). Frequency dependence of tunability in stepped, graded, and PMNT70-30 films.

\section{Capacitance-voltage $(C-V)$ and hysteresis $(P-E)$ measurements}

The room-temperature $C-V$ measurements of the graded, stepped, and PMNT70-30 films at $100 \mathrm{kHz}$ are shown in Fig. 6(a). The $C-V$ of graded films showed a higher nonlinear behavior compared to that of the other stepped and single layer compositions. In any of the general ferroelectric films, the $C-V$ curves show a butterfly kind of behavior where this asymmetry could be attributed to many reasons such as domain-wall pinning and asymmetric electrodes. In the case of the stepped and graded configurations the asymmetry is an inherent property due to the asymmetry in the band structure seen by the top electrode and the bottom electrode. ${ }^{30}$ The loss factor $D$ of the graded films showed a similar characteristic with a room-temperature value of about 0.05 at $100 \mathrm{kHz}$. The tunability of the films was calculated using the formula given below.

$$
\text { Tunability }=\frac{\varepsilon(T, 0)-\varepsilon(T, E)}{\varepsilon(T, 0)} 100 \% .
$$

The tunability of the three different films at $100 \mathrm{kHz}$ measured at room temperature is shown in Fig. 6(b). The tun- 
abilities of the graded, stepped, and PMT70-30 at $100 \mathrm{kHz}$ were found to be $59 \%, 49 \%$, and $32 \%$, respectively. The graded film was found to have the highest tunability among the three films. Such high tunability could be due to the randomly oriented polar clusters, which act as the domains and the separation between these clusters acting as a domain wall. ${ }^{31}$ Statistically the tunability is dominated by the distribution of nanopolar regions across the film due to the variation of lead titanate. This distribution of the density of the polar clusters across the film which in turn gives rise to a high chemical heterogeneity within the system could be responsible for the high tunability behavior. The existence of polar clusters and their dynamics was found to be similar to that of domains by means of ergodic behavior of those polar clusters in the case of normal ferroelectrics. ${ }^{4}$ In the case of both stepped and graded ferroelectrics, there is an existence of both ergodic and nonergodic polar clusters even at room temperature due to the spatial fluctuation of the composition. The frequency dependence of the tunability of the fabricated thin films is shown in Fig. 6(c), which shows that the tunability is almost constant with frequency. Apart from the high tunability observed, the graded and stepped films showed a weak ferroelectricity at room temperature, which was also confirmed from the polarization hysteresis measurements.

The polarization hysteresis $(P-E)$ measurements of both stepped and graded films were carried out at different temperatures. Figure 7(a) shows the polarization hysteresis curve of a stepped film, which exhibited a narrow slim loop behavior, and is a characteristic of a normal relaxor material with a single composition. The stepped films showed a saturation polarization of around $40 \mu \mathrm{C} / \mathrm{cm}^{2}$. The loop was found to be very slim than that of the single-layer composition shown in Fig. 7(c), which may be due to the pinning of the polar clusters in the strain field present at the interfaces of the layers. No significant field-induced widening of the hysteresis loop was found and hence only two measurements are shown for discussion. Figure 7(b) shows the polarization hysteresis of a graded film at different voltages, which exhibited slim loop characteristics similar to that of a single layer. The saturation polarization was found to be around $40 \mu \mathrm{C} / \mathrm{cm}^{2}$ in these films and the loop was found to be wider than that observed in the case of stepped films. PMNPT70-30 composition is known to have a superior property among the selected compositions and hence only that particular composition is used for comparison. ${ }^{7,8}$

\section{CONCLUSIONS}

Thin films of different configurations in terms of composition as explained earlier were fabricated using pulsed laser ablation technique and their dielectric phase transition, capacitance-voltage, and polarization hysteresis studies were carried out. The compositional variation of both step and graded configurations was confirmed through SIMS analysis of individual elements across the thickness. The phase transition of the stepped films was found to have an individual layer dominance at a given temperature window and the influence of those individual layer contributions to the phase transition is seen clearly from the local maxima observed in

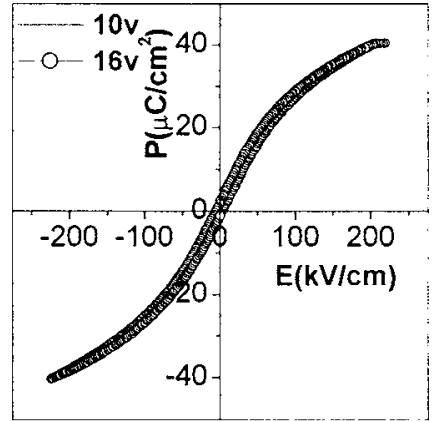

(a)
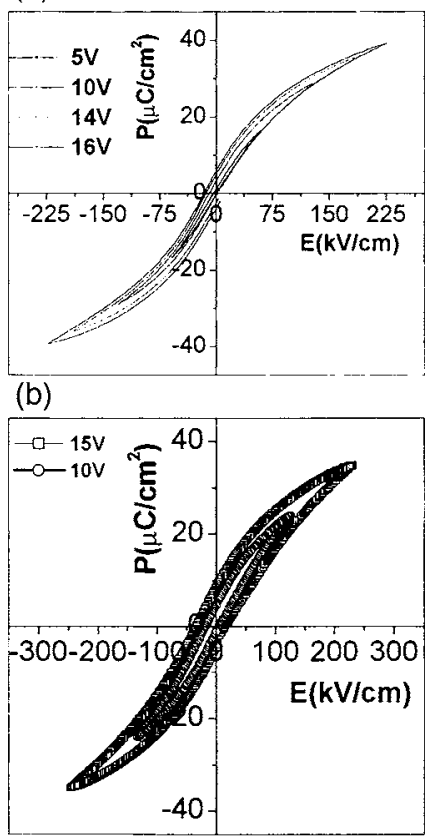

(c)

FIG. 7. (a) Polarization hysteresis of a stepped film. (b) Polarization hysteresis of a graded film. (c) Polarization hysteresis of a PMNT70-30 thin film.

the phase-transition curve. In the case of the graded films the phase transition was found to be a combinatorial effect of all the composition fluctuation introduced by means of the distribution of composition of PT. The dielectric constant was observed to have an ensemble-averaged value with temperature in the case of the graded films. Hence, the modified Curie-Weiss plot was used to characterize the diffusiveness and the value of $\gamma$ was found to be 2.43 at $100 \mathrm{kHz}$. The Curie constant was found to be $1.05 \times 10^{5}$. This shows the enhancement in the diffusiveness of the phase transition, whereas the normal relaxor ferroelectrics with a single composition would have a $\gamma$ value of 2 . The order parameter $[q(T)]$ which is a measure of the fraction of polar clusters present was calculated at different temperatures at given frequencies. The $q(T)$ had a nonzero value for temperatures well above the transition temperatures which showed the existence of the polar clusters well above the transition temperature $(370 \mathrm{~K})$ found by extrapolating the Curie-Weiss plot to the $x$ axis. A high nonlinear behavior was observed in the case of capacitance with voltage and hence the tunability of the graded films was found to be higher than the stepped and the single compositional layers. The high tunability is attributed to the existence of the nanopolar clusters in the 
system due to the increase in the chemical heterogeneity across the film apart from which is present in the relaxor films naturally. The high-frequency tunability of the graded film was found to be twice that of the single-compositioned films. Polarization hysteresis measurements showed a slim loop behavior both in the case of stepped and graded films with a saturation polarization value of $40 \mu \mathrm{C} / \mathrm{cm}^{2}$. This slim loop behavior is known to be suitable for the solid-state actuator applications. ${ }^{5}$

${ }^{1}$ J. V. Mantese, N. W. Schubring, A. Micheli, and A. B. Catalan, Appl. Phys. Lett. 67, 721 (1995).

${ }^{2}$ J. V. Mantese, N. W. Schubring, A. Micheli, A. B. Catalan, M. S. Mohammad, B. Naik, and G. W. Auner, Appl. Phys. Lett. 71, 2047 (1997).

${ }^{3}$ L. J. Kim, D. Jung, J. Kim, Y. S. Kim, and J. Lee, Appl. Phys. Lett. 82, 2118 (2003).

${ }^{4}$ S. G. Lu, X. H. Zhu, C. L. Mak, K. H. Wang, H. L. W. Chang, and C. L. Choy, Appl. Phys. Lett. 82, 2877 (2003).

${ }^{5}$ K. Uchino, Piezoelectric Actuators and Ultrasonic Motors (Kluwer Academic, Dordrecht, 1996), and references therein

${ }^{6}$ T. R. Shrout and J. F. Fielding, Jr., Proc.-IEEE Ultrason. Symp., 711 (1990).

${ }^{7}$ S. W. Choi, T. R. Shrout, S. J. Jang, and A. Bhalla, Ferroelectrics 100, 29 (1989).

${ }^{8}$ S. W. Choi, T. R. Shrout, S. J. Jang, and A. Bhalla, Mater. Lett. 8, 253 (1989).

${ }^{9}$ L. E. Cross, Ferroelectrics 76, 241 (1987).

${ }^{10}$ L. E. Cross, Ferroelectrics 151, 305 (1987), and references therein.

${ }^{11}$ P. K. Davies and M. A. Akbas, J. Phys. Chem. Solids 61, 154 (2000).
${ }^{12}$ G. Burns and F. H. Dayol, Ferroelectrics 104, 25 (1990).

${ }^{13}$ D. Vieland, S. J. Jang, L. E. Cross, and H. Wuttig, J. Appl. Phys. 68, 2916 (1990).

${ }^{14}$ N. de Mathan, E. Husson, G. Galvarin, J. R. Gavani, A. W. Hewat, and A. Morell, J. Phys.: Condens. Matter 3, 8159 (1991).

${ }^{15}$ G. A. Smolensky and A. I. Agranovskaya, Sov. Phys. Solid State 1, 1429 (1959).

${ }^{16}$ S. N. Dorogstsev and N. K. Yushin, Ferroelectrics 112, 27 (1990).

${ }^{17}$ D. Viehland, S. J. Jang, L. E. Cross, and M. Wuttig, J. Appl. Phys. 68, 2916 (1990).

${ }^{18}$ V. Westpal, W. Kleeman, and M. D. Glinchuk, Phys. Rev. Lett. 68, 847 (1992).

${ }^{19}$ A. E. Glazounov, A. K. Taganstev, and A. J. Bell, Phys. Rev. B 53, 11281 (1996).

${ }^{20}$ R. Pirc and R. Blinc, Phys. Rev. B 60, 13470 (1990).

${ }^{21}$ S. Swartz and T. Shrout, Mater. Res. Bull. 17, 1245 (1982).

${ }^{22}$ A. Laha, S. Saha, and S. B. Krupanidhi, Thin Solid Films 424, 274 (2003).

${ }^{23}$ C. B. Sawyer and C. H. Tower, Phys. Rev. 35, 269 (1930).

${ }^{24}$ K. H. Chew, L. H. Ong, J. Osman, and D. R. Tilley, Appl. Phys. Lett. 77, 2755 (2000)

${ }^{25}$ C. Wang and D. R. Tilley, Solid State Commun. 118, 333 (2001).

${ }^{26}$ M. Tyunina and J. Levoska, Phys. Rev. B 63, 224102 (2001).

${ }^{27}$ J. V. Mantese, N. W. Schubring, A. Micheli, M. P. Thompson, R. Naik, G. W. Auner, I. B. Misirlioglu, and S. P. Alpay, Appl. Phys. Lett. 81, 1068 (2002).

${ }^{28}$ B. E. Vugmeister and H. Rabitz, Phys. Rev. B 57, 7581 (1998).

${ }^{29}$ B. E. Vugmeister and H. Rabitz, Phys. Rev. B 61, 14448 (2000).

${ }^{30}$ S. J. Lee, S. E. Moon, H. C. Ryu, M. H. Kwak, Y. T. Kim, and S. K. Han, Appl. Phys. Lett. 82, 2133 (2003).

${ }^{31}$ M. Abplanalp, D. Barosova, P. Bridenbaugh, J. Erhart, J. Fousek, P. Gunter, J. Nosek, and M. Sulc, J. Appl. Phys. 91, 3797 (2002). 